\title{
O Reconhecimento Facial como uma das Vertentes da Inteligência Artificial (IA): um estudo de prospecção tecnológica
}

\author{
Facial Recognition as one of the Artificial Inteligence Topics: a study of \\ technological prospection
}

\author{
Valdir Silva Conceiçãó ${ }^{1}$ \\ Edna Maria Nunes ${ }^{1}$ \\ Angela Machado Rocha ${ }^{1}$ \\ ${ }^{1}$ Universidade Federal da Bahia, Salvador, BA, Brasil
}

\begin{abstract}
Resumo
A prospecção tecnológica é um instrumento utilizado para verificar e mapear o desenvolvimento tecnológico e científico e serve para influenciar e direcionar a inovação na indústria, na sociedade e na economia de forma que os inventores não se atenham a trabalhar em produtos que já tenham sido gerados e protegidos, também muito utilizada para melhorar o que já existe em termos tecnológicos. Este trabalho revisou a literatura referente ao reconhecimento facial, tendo como base os pedidos de patentes nos escritórios. A metodologia utilizada foi a prospecção tecnológica patentária, que teve como ferramenta de pesquisa a plataforma Orbit. Neste estudo foram encontradas 17.050 patentes, e a maior parte delas esta na classificação CPC como G - Física, com 78,92\%, e, desse total, 80,20\% estão no CPC G06 - Cômputo, cálculo; contagem, sendo que 53,81\% se relacionam ao código CPC G06K referente à identificação de dados; apresentação de dados; suporte de dados; manipulação de transporte de dados.
\end{abstract}

Palavras-chave: Prospecção. Reconhecimento Facial. Patente.

\begin{abstract}
Technological prospection is an instrument used to verify and map technological and scientific development and serves to influence and direct innovation in industry, society and the economy so that inventors do not focus on working on products that have already been generated. and protected, and also serve to improve what already exists in technological terms. The objective of the present work was to map the registered research regarding facial recognition, based on patent applications in the offices. The methodology used was the patenting technological prospection, using the Orbit platform as a tool. In this study, 17050 patents were found. Most patents are in CPC classification as $\mathrm{G}$ - Physics with $78.92 \%$ and of this total $80.20 \%$ are in CPC G06 - Calculation, calculation; $53.81 \%$ are related to $\mathrm{CPC}$ code $\mathrm{G} 06 \mathrm{~K}$ regarding data identification; data presentation; data support; data transport handling.
\end{abstract}

Keywords: Prospecting. Facial Recognition. Patent.

Área Tecnológica: Engenharia Industrial. Computação. Segurança Pública. Recursos Humanos. 


\section{Introdução}

O desenvolvimento tecnológico avança em ritmo acelerado no mundo globalizado, principalmente devido às pesquisas mundiais diante das necessidades humanas. Um dos maiores avanços tecnológicos nos últimos anos foi a expansão da Inteligência Artificial (IA) (FIGLIUZZI, 2018).

A tecnologia de IA, a digitalização, o sensoriamento, a impressão 3D e a mecanização que converge com as tecnologias digitais, físicas e biológicas são denominadas a Quarta Revolução Industrial (PORFÍRIO, 2018).

A IA é um ramo recente da ciência e da engenharia originária da década de 1950 e que tem o objetivo de analisar e de interpretar os dados complexos, simular ou reproduzir a inteligência humana em máquina e traz como resultados o diagnóstico, o tratamento e a previsão de resultados (WELCHEN, 2019).

A IA migrou da ficção científica reproduzida em filmes, passou para a fase de experimentos em laboratórios e, nos tempos atuais, realiza operações reais nos negócios. Ela pode ser observada em diversos campos, como na previsão de demanda por produtos, carros inteligentes, Data Security (segurança de dados na internet), financial trading (mercado financeiro), saúde, agricultura, indústria, personalização de marketing, prevenção de fraude, recomendações, Natural Language Processing (NLP), reconhecimento facial, entre outros (BORINI, 2017).

O uso da IA está modificando o dia a dia das pessoas e tende a transformar as estratégias de marketing em decorrência das mudanças observadas no ser humano, influenciando os modelos de negócio e os processos de venda e de atendimento ao cliente (DAVENPORT et al., 2019).

Um dos segmentos que acompanham essa evolução é o sistema de reconhecimento facial que permite a identificação de pessoas em meio à multidão, sendo aplicado na área de segurança pública e privada e no desbloqueio dos aparelhos digitais. Além da biometria, outras formas utilizadas de reconhecimento do ser humano são a íris, a retina, as digitais dos dedos das mãos, as veias da mão, a voz, o cheiro, o rosto, entre outros (KOCH, 2012).

O rosto humano apresenta características variáveis em cada pessoa, porém existem combinações básicas que não se alteram, tornando-se pontos comuns como os dois olhos e a distância entre eles, o nariz e o seu comprimento, a bochecha, a boca e o queixo, que podem ser lidos por softwares por meio de algoritmos gravados e armazenados em bancos de dados que têm como princípio detectar as formas geométricas da face, montar o quebra-cabeça e, posteriormente, fazer comparações com as fotos existentes em bancos de dados (KOCH, 2012; BATISTA et al., 2017).

A biometria, por exemplo, é uma palavra de origem grega bios (vida) metron (medida), nela são realizados estudos com a utilização da estatística do comportamento e de qualidades físicas dos indivíduos, sendo amplamente usada como uma ferramenta para o controle de segurança (KOCH, 2012).

O reconhecimento facial por meio de computador vem sendo pesquisado há mais de 40 anos (ACQUISTI; GROSS; STUTZMAN, 2014). Segundo a consultora MarketsandMarkets (2018), o mercado mundial de reconhecimento facial movimenta US\$ 3,3 bilhões, e a previsão para 2020 é de que esse valor seja equivalente a US\$ 7,7 bilhões (ELOLA, 2018). 
Existem algumas questões desfavoráveis ao uso do reconhecimento facial, por exemplo, o seu uso indevido pelas organizações, a possibilidade de o sistema ser hackeado e pessoas desonestas obterem dados sigilosos, o uso indiscriminado por governos totalitários para ameaçar os cidadãos rivais, a possibilidade de comercialização dos dados obtidos, a repressão a grupos que não coadunem com a política implantada por certos governos e a invasão da privacidade do indivíduo. Como fator positivo, é possível citar o aumento da segurança pública, possibilitando que as autoridades competentes encontrem pessoas desaparecidas, criminosas ou em situações suspeitas, entre outros, retirando das ruas as que possuem pendências judiciais, de forma que essa atitude tem o potencial de evitar que esses maus elementos cometam novos delitos contra a sociedade.

O reconhecimento facial foi utilizado no carnaval de Salvador em 2019, retirando de circulação pessoas que estavam em débito com a justiça. Essa ferramenta também foi utilizada no carnaval do Rio de Janeiro e na Copa América (VETTORAZZO; PITOMBO, 2019).

Pretende-se neste trabalho realizar uma prospecção tecnológica das patentes existentes e suas inovações para o reconhecimento facial, além de identificar os avanços tecnológicos surgidos ao longo dos anos e as perspectivas futuras. Nesse contexto, os avanços nos estudos de prospecção tecnológica vêm contribuindo consideravelmente na atualidade e na orientação para desenvolvimento futuro.

\section{Metodologia}

A metodologia de pesquisa utilizada foi a exploratória tomando como base as informações em sítios, teses, dissertações, artigos científicos e monografias relacionadas ao tema, demonstrando o caráter bibliográfico referenciado em Lakatos e Marconi (1991).

Conforme aponta Gil (2010, p. 41), “[...] a pesquisa exploratória visa proporcionar maior familiaridade com o problema, com vistas a torná-lo mais explícito ou a constituir hipóteses, sendo isso feito ao longo do trabalho". Gil (2010, p. 42) menciona ainda que "[...] as pesquisas descritivas identificam as características de determinada população ou fenômeno".

A prospecção tecnológica patenteária sobre IA, especificamente reconhecimento facial, foi realizada utilizando-se os pedidos de patentes depositados em mais de 90 países, tendo como ferramenta de pesquisa a plataforma Orbit, que é um software de negócios que faz pesquisas, seleção, análise e exportação de informações contidas em patentes, com cobertura geográfica em quase uma centena de países e de autoridades de patentes.

A pesquisa foi realizada em agosto de 2019 e foram utilizados como palavras-chave artificial intelligence, facial recognition and intelligence, facial recognition and artificial, facial recognition, facial and recognition e face and recognition. Foram considerados válidos todos os documentos que apresentassem a combinação do termo reconhecimento facial e inteligência artificial no título e no resumo.

A análise documental gerada consistiu na avaliação da distribuição dos depósitos de patentes por país, depósitos por inventor e por ano. Foram analisados todos os pedidos de patentes existentes até o dia 3 de agosto de 2019. 


\section{Revisão da Literatura}

O desenvolvimento do estudo sobre Inteligência Artificial começou após a Segunda Guerra Mundial e foi publicado em artigo do matemático Alan Turing, cujo título é Computing Machinery and Intelligence. O seu nome foi sugerido por volta de 1956 por John McCarthy, um professor universitário, em uma conferência de especialistas denominada "O Eros Eletrônico", que ocorreu nas instalações do Darmouth College (COSSETI, 2018).

A Inteligência Artificial pode ser agrupada em duas dimensões e em quatro categorias:

Quadro 1 - Agrupamento da IA

\begin{tabular}{|c|c|c|c|}
\hline \multicolumn{4}{|c|}{ CATEgorias } \\
\hline \multirow{3}{*}{ 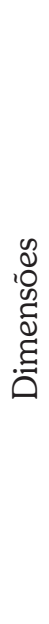 } & & Humano & Racional \\
\hline & 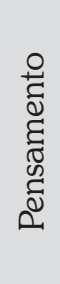 & $\begin{array}{l}\text { Sistemas que pensam como humanos } \\
\text { (abordagem da modelagem cognitiva) } \\
\text { Automação das atividades inerentes ao ser } \\
\text { humano, atividades como tomada de decisão, } \\
\text { resolução de problemas (BELLMAN, 1978). } \\
\text { Exemplo: redes neurais artificiais. }\end{array}$ & $\begin{array}{c}\text { Sistemas que pensam racionalmente } \\
\text { (abordagem das leis do pensamento) } \\
\text { Estudo das faculdades mentais por } \\
\text { meio de modelos computacionais } \\
\text { (CHARNIAK; MCDERMOTT, 1985). } \\
\text { Estudos das operações que fazem possivel } \\
\text { perceber, raciocinar e atuar (WISTON, 1992). }\end{array}$ \\
\hline & 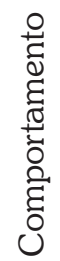 & $\begin{array}{c}\text { Sistemas que agem como humanos } \\
\text { (abordagem do Teste de Turing) } \\
\text { A arte de criar máquinas que realizam funções } \\
\text { que requerem inteligência quando realizadas } \\
\text { por humanos (KURZWEIL, 1990). } \\
\text { Exemplo: robôs. }\end{array}$ & $\begin{array}{l}\text { Sistemas que agem racionalmente } \\
\text { (abordagem de agentes racionais) } \\
\text { Preocupação com a automação do } \\
\text { comportamento inteligente (LUGER; } \\
\text { STUBBLEFIELD, 1993). }\end{array}$ \\
\hline
\end{tabular}

Fonte: Russel e Norvig (1995) e Alves (2000)

A IA se refere a programas, logaritmos, sistemas e máquinas que atuam de forma similar aos humanos e imitam parte do seu comportamento por meio de uso de várias tecnologias empregadas para a aprendizagem das máquinas, de forma a desempenhar atividades com pouca ou nenhuma interferência das pessoas. A análise feita pela IA pode ser realizada com dados não numéricos, como texto, voz, imagem e face (DAVENPORT et al., 2019).

As pessoas têm certa preocupação com relação ao uso dos dados pelas empresas, principalmente no que se refere ao uso indevido e quando existe a combinação da IA com a Big Data, já que, nesse caso, há uma quebra da privacidade desses dados coletados sem o consentimento prévio do indivíduo. Existe uma falta de regulamentação sobre o uso dos dados que podem ser obtidos por hackers, já que não há nenhum sistema perfeito e à prova de violações do sistema. Além disso, ressalta-se a questão da falta de confiabilidade das pessoas em geral nas organizações, que podem fazer uso indevido ou até vender os dados para quem se interessar em ter um banco de dados, o que fere a ética (FELDSTEIN, 2019).

O reconhecimento facial é uma ferramenta utilizada para identificar ou verificar pessoas, tendo como instrumento a face, quando captura, analisa e compara com padrões detalhados as características faciais de um indivíduo. Nos dias atuais, essa ferramenta é considerada o meio mais natural de medição da face de uma pessoa por ser invasivo. Faz a comparação do rosto baseado na geometria facial, incluindo a distância e a proporção entre os olhos e as sobran- 
celhas, comprimento da linha da mandíbula, tamanho do crânio, linha do cabelo, largura do nariz, da boca, dos lábios, entre outras 80 bases faciais ou pontos nodais.

Possui três categorias distintas:

a) Detecção de Rosto - diferencia o rosto de um objeto; não identifica um rosto como pertencente a um indivíduo particular; ajuda a contar e a analisar fluxo de pessoas; estima tamanho da população.

b) Autenticação Facial - compara os recursos de uma imagem de rosto com os armazenados em um único perfil armazenado anteriormente; mapeia os recursos do rosto de um indivíduo para fins de autenticação "1 para 1".

c) Correspondência Facial - tenta corresponder um rosto usando recursos de uma imagem de rosto com os armazenados em um banco de dados; mapeia os recursos do rosto de um indivíduo para fins de correspondência "1 para muitos" (FELDSTEIN, 2019).

Existem problemas relacionados à interpretação dos dados, que podem provocar erros de identificação de indivíduos, e, nesse caso, o mais correto é evitar o julgamento das pessoas em decorrência de equívocos no reconhecimento facial. Há também o perigo do uso da tecnologia por governantes, com o objetivo de impor restrições à liberdade individual e de repressão política contra os desafetos (FELDSTEIN, 2019).

A prospecção tecnológica é uma ferramenta que busca a inovação para que as organizações se diferenciam das similares, antecipa tendências e mudanças que permitam se posicionar em condições favoráveis, evita que se desenvolvam invenções que já estão registradas e consolidadas e que permite a monitoração de cenários entre outras servidões (RIBEIRO, 2018).

Existem diversas formas de captação da prospecção tecnológica, com várias fontes de informações e técnicas para o tratamento dos dados obtidos na busca e, também, para a geração de resultados, de acordo com as abordagens e os enfoques direcionados aos dados (RIBEIRO, 2018).

A prospecção possui quatro fases distintas, iniciando pela preparação, visando à definição dos objetivos e da metodologia, que deve ser clara; posteriormente ocorre a fase pré-prospectiva, na qual são definidos e detalhados a metodologia e o levantamento da fonte de dados a ser utilizada; a terceira fase é da coleta, análise e tratamento dos dados obtidos e, por último, faz-se a comunicação dos resultados, em conjunto com a implementação das ações a serem tomadas (MAYERHOFF, 2008).

Todos os pedidos de patentes são classificados na área tecnológica à qual pertencem. Existe a Classificação Internacional de Patentes (IPC) desde 1971, que foi oriunda do Acordo de Estrasburgo e representa o sistema de classificação internacional de patentes, cujas áreas tecnológicas são divididas em oito seções, variando de A a H. Essa classificação tem o intuito de facilitar a identificação das patentes que têm a mesma área de conhecimento e interesse tecnológico e a investigação da técnica da arte (PRIESNITZ et al., 2015; INPI, 2019).

As classificações das seções são as seguintes: A - Necessidades Humanas; B - Operações de Processamento; Transporte; C - Química e Metalurgia; D - Têxteis e Papel; E - Construções Fixas; F - Engenharia Mecânica; Iluminação; Aquecimento; Armas; Explosão; G - Física; e H - Eletricidade (WIPO, 2019). 
Também existe a Classificação Cooperativa de Patentes (CPC) desde 2014, que foi criada pela EPO/USTO e que tem como base o IPC, porém, com mais detalhes, possuindo cerca de 200 mil grupos. É um sistema muito utilizado pela Comunidade Europeia (INPI, 2019).

\section{Resultados e Discussão}

Na pesquisa foi possível identificar o desenvolvimento tecnológico na área de IA focando no reconhecimento facial.

O Quadro 2 mostra o escopo de busca utilizado para a prospecção tecnológica e o número de patentes depositadas nos bancos de dados, incluindo as ativas, as vencidas, as pendentes $e$ as revogadas. $\mathrm{O}$ parâmetro de busca foram as palavras-chave: recognition, facial, recognition or facial, facial and recognition, facial and image, face and person e face and recognition, obtendo-se o seguinte resultado:

Quadro 2 - Quantitativo de patentes depositadas

\begin{tabular}{|c|c|c|c|c|c|c|c|}
\hline \multirow[b]{2}{*}{ Palavra-Chave } & \multirow[b]{2}{*}{ TOtaL } & \multicolumn{6}{|c|}{ IPC } \\
\hline & & $\begin{array}{l}\text { G06K- } \\
009 / 00\end{array}$ & $\begin{array}{l}\text { G06K- } \\
009 / 62\end{array}$ & $\begin{array}{l}\text { G06T- } \\
007 / 00\end{array}$ & $\begin{array}{l}\text { G06T- } \\
001 / 00\end{array}$ & $\begin{array}{l}\text { G06F- } \\
017 / 30\end{array}$ & Quantitativo \\
\hline Face and recognition & 59.830 & 17.195 & 4.181 & 2.901 & 2.342 & 1.743 & 28.362 \\
\hline Facial and recognition & 17.050 & 8.744 & 2.198 & 1287 & 864 & 902 & 13.995 \\
\hline Facial recognition & 6.686 & 3.249 & 733 & 441 & 238 & 460 & 5.121 \\
\hline $\begin{array}{l}\text { Facial recognition } \\
\text { and intelligence }\end{array}$ & 398 & 153 & 25 & 4 & 2 & 18 & 202 \\
\hline $\begin{array}{l}\text { Facial recognition } \\
\text { and artificial }\end{array}$ & 209 & 121 & 31 & 12 & 5 & 15 & 184 \\
\hline $\begin{array}{l}\text { Facial recognition and } \\
\text { artificial intelligence }\end{array}$ & 120 & 66 & 15 & 2 & 2 & 8 & 93 \\
\hline Total & 84.293 & 29.528 & 7.183 & 4.647 & 3.453 & 3.146 & 47.957 \\
\hline
\end{tabular}

Fonte: Elaborado pelos autores deste artigo baseado na ferramenta Orbit (2019)

Observa-se no Quadro 2 que quanto maior a depuração dos termos, maior é a tendência a uma redução substancial do quantitativo de patentes. Dessa forma, como critério de seleção de dados para o estudo da prospecção, foram utilizadas as seguintes palavras-chave: Facial and Recognition.

A data do primeiro registro do reconhecimento facial foi em 29 de agosto de 1972. A Patente n. 1.972 GB-0039965 depositada nesse ano teve como título Data printing system (Sistema de impressão de dados), que representava um produto tecnológico como um sistema automático para analisar e registar independentemente os limites dos detalhes da imagem e da distribuição de cores de cenas policromadas particulares. O sistema produzia superfícies de trabalho esquematizadas nas quais pessoas relativamente desleixadas podiam criar renderizações de uma cena inicial, por exemplo, uma cena gravada pela primeira vez num filme fotográfico. A descrição 
temporal do número de registros de patentes nos últimos 25 anos encontra-se no Gráfico 1, que mostra a quantidade de patentes concedidas em cada ano.

Gráfico 1 - Evolução das patentes relacionadas ao desenvolvimento de inovações tecnológicas na área de reconhecimento facial nos últimos 25 anos

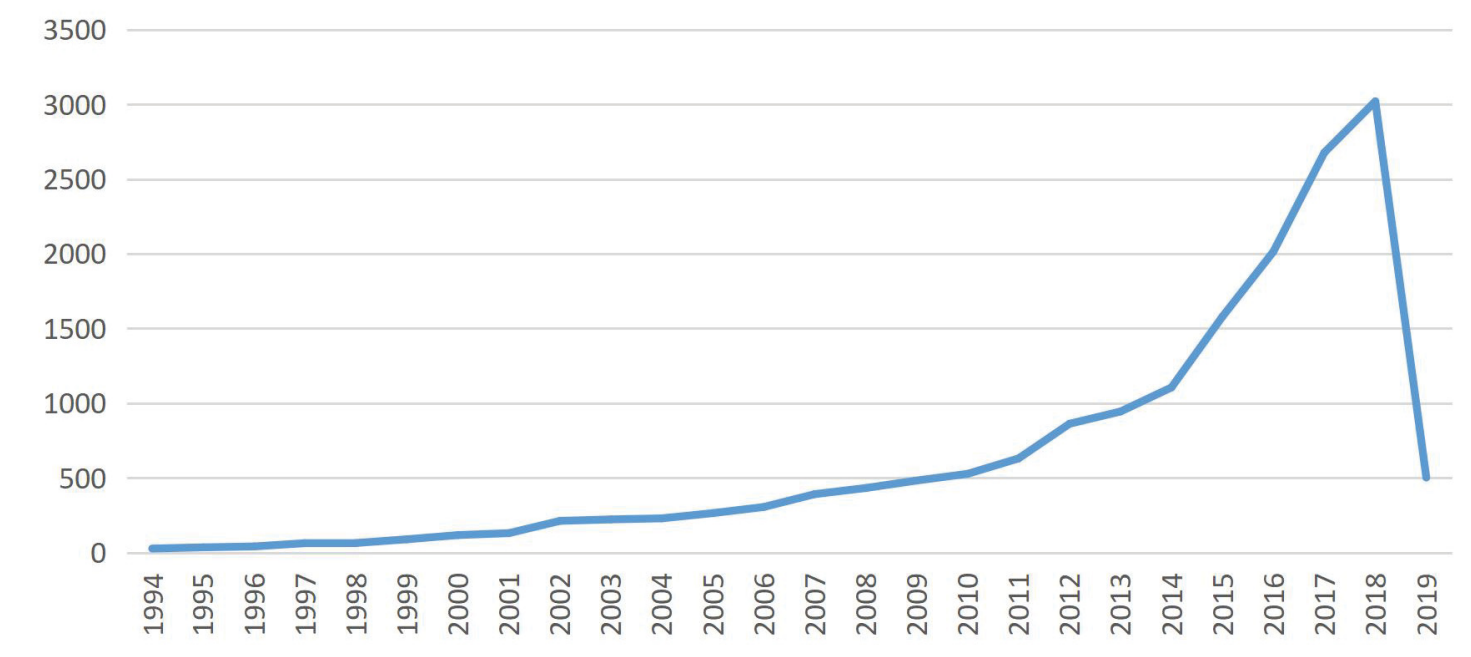

Fonte: Elaborado pelos autores deste artigo com base na ferramenta Orbit (2019)

O Gráfico 1 mostra a linha cronológica das patentes concedidas nos últimos 25 anos, entre 1994 e 2019, com um crescimento exponencial a partir de 2014, que pode estar associado a um aumento de uso de dispositivos conectados com essa tecnologia, por exemplo, os dispositivos móveis. Nos anos anteriores a 1994, o crescimento era próximo do linear, sem um crescimento significativo, começando a ter uma substancial importância a partir do ano 2000. A legislação de registro de patentes prevê seu sigilo durante 18 meses, o que acaba por impactar no resultado dos anos de 2018 e 2019. Portanto, nesses anos, o resultado pode não corresponder à realidade em termos de registro de patentes, principalmente com a redução drástica ocorrida em 2019 e mostrada no Gráfico 1.

Na década de 1970, as patentes tinham as seguintes tecnologias: a impressão de dados (1); leitura ótica para scanner (1); e segmento de saúde (2). Na década de 1980, foram voltadas para a área de comunicação e imagem (7), extração de recursos (4), para instrumentos (3), expressão (2), de caracteres (3), reconhecimento facial (2), de presença (2), para restauração facial (1), leitor ótico (1), para jogo (1), entre outros

Na comparação dos últimos 25 anos, o maior crescimento deu-se em 2002 com 63,08\%, e o menor percentual foi em 1998 com 1,59\% de aumento de registro de patentes. A média de aumento de depósitos a partir de 1972 até 2018 ficou em torno de 32,04\%. Em 2010, o investimento mundial em P\&D foi de US\$ 508 bilhões, enquanto em 2015 foi de US\$ 679,8 bilhões, sendo que, para produtos do segmento eletrônico, esse valor foi de US\$163 bilhões. $\mathrm{O}$ fator que influenciou esse investimento foi o de smartphone, com a Samsung, a maior desenvolvedora de tecnologia do segmento de reconhecimento facial, investindo US $\$ 12,7$ bilhões. Os setores de software e de computação gastaram US\$ 88 bilhões em 2016. Em 2018, a Samsung investiu cerca de 22 bilhões em IA (SAMSUNG, 2018). 
Neste estudo, percebeu-se que o código mais citado foi o da classe G06, que corresponde aos simuladores utilizados para o método de cálculo mais avançado e o funcionamento de aparelhos ou de um sistema, abrangendo também o processamento de dados de imagens ou a sua geração, como é o caso do reconhecimento facial.

A subclasse G06K tem como objetivo a identificação de caracteres ou de dados, como é o caso da face das pessoas, com apresentação visual ou outra forma de dados identificados.

Gráfico 2 - Número de patentes relacionadas ao desenvolvimento de inovações tecnológicas na área de reconhecimento facial, pela Classificação CPC

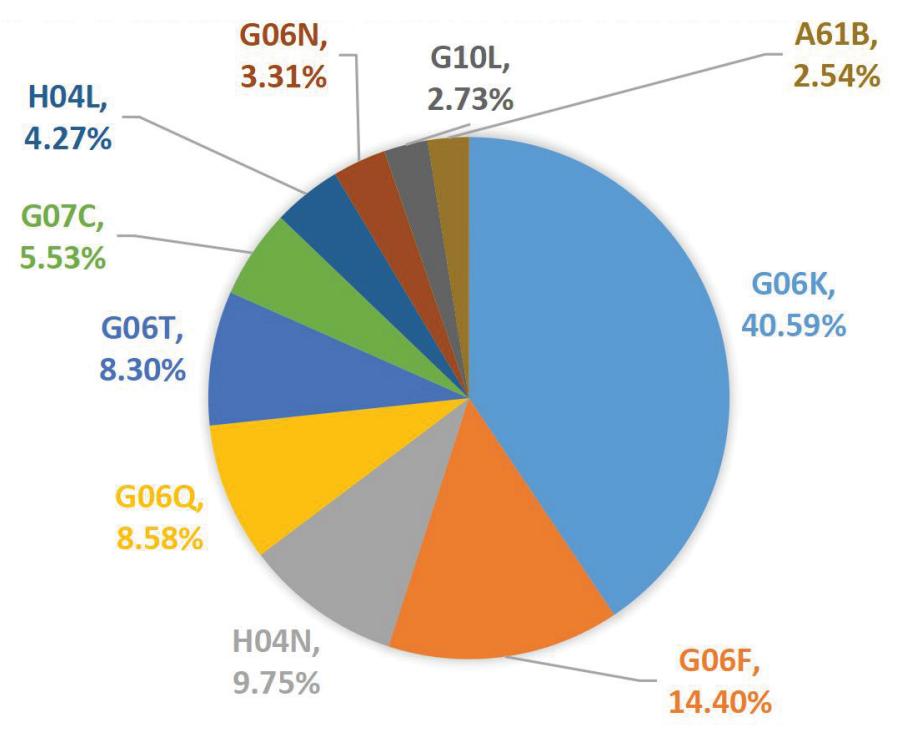

Fonte: Elaborado pelos autores deste artigo com base na ferramenta Orbit (2019)

O Código CPC descreve as seguintes subclasses em relação ao resultado encontrado no Gráfico 2:

G06K - Reconhecimento de dados; apresentação de dados; transportadores de registros; manuseando transportadores.

G06F - Processamento de dados digitais elétricos.

H04N - Comunicação pictral.

G06Q - Sistemas ou métodos de tratamento de dados, especialmente adaptados para fins administrativos, comerciais, financeiros, gerenciais, de supervisão ou de previsão; sistemas ou métodos adaptados especialmente para fins administrativos, comerciais, financeiros, gerenciais, de supervisão ou de previsão, não fornecidos de outra forma

G06T - Processamento ou geração de dados de imagem, em geral.

G07C - Registro de horário ou de atendimento; aparelhos de votação ou loteria.

H04L - Transmissão de informações digitais.

G06N - Sistemas de computador baseados em modelos computacionais específicos.

G10L - Reconhecimento da fala; processamento de voz ou voz

A61B - Diagnóstico, cirurgia; identificação. 
O Gráfico 2 mostra a classificação G, referente à Física, que representa 83,44\% das patentes depositadas. Uma grande parcela das patentes depositadas é da família G06K, que representa $40,59 \%$ das parcelas, seguida da classificação G06F com 14,40\% dos registros das patentes.

De acordo com o IPC, o maior contingente é o código G06K 009/00, com 8.744 registros, que está relacionado com os métodos ou disposições de leitura ou identificação de caracteres impressos ou escritos ou de identificação de padrões.

A Figura 1 mostra a distribuição das patentes depositadas nos países.

Figura 1 - Distribuição dos registros de depósito de patentes relacionadas ao desenvolvimento de inovações tecnológicas na área de reconhecimento facial por países

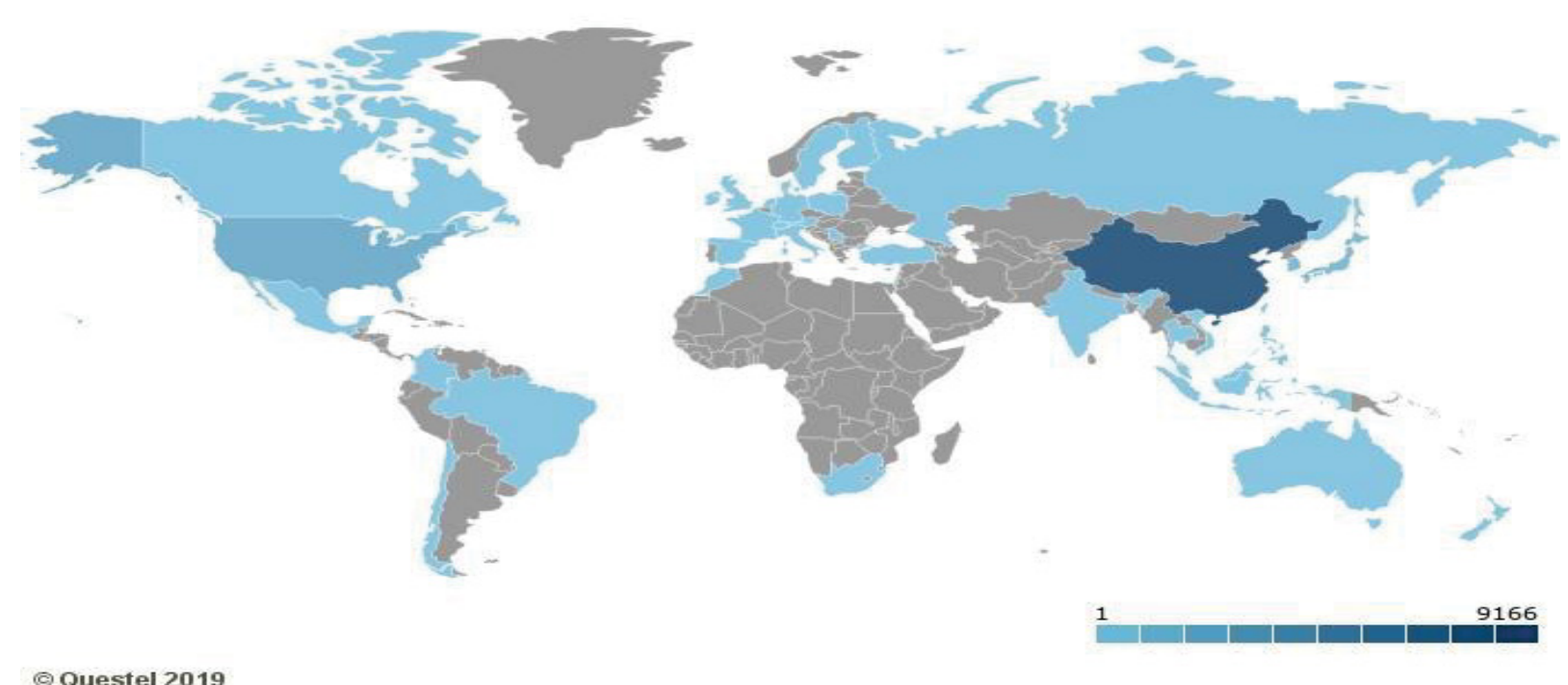

Fonte: Orbit (2019)

Em relação ao total de patentes depositadas, a China lidera com 9.166 depósitos, o que equivale a 47,69\% do total dos depósitos, seguida de longe pelos Estados Unidos com 2.660 depósitos, o que equivale a $13,84 \%$ do total dos depósitos. O Brasil possui 131 patentes depositadas, equivalendo a $0,68 \%$ do total dos depósitos.

O resultado mostra o interesse dos inventores chineses e estadunidenses pelo uso da tecnologia de reconhecimento facial. Essa condição de liderança, tem potencial de gerar divisas para o país na venda de produtos com essa tecnologia. Também tem o potencial de gerar receitas para o detentor da patente e divisas para o país, além de demonstrar que esse resultado é fruto de investimento em PD\&I, gerando novas oportunidades para incentivar a inovação nas microempresas (SANTOS et al., 2015).

Os maiores inventores são chineses, o que é coerente com o quantitativo de patentes depositadas no país, como pode ser visto no Gráfico 3. 
Gráfico 3 - Inventores de patentes relacionadas ao desenvolvimento de inovações tecnológicas na área de reconhecimento facial

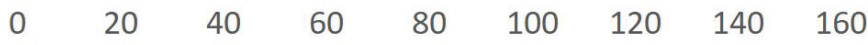

THE INVENTOR HAS WAIVED THE RIGHT TO BE...

SUN LIHUA,

ZHANG HUILIN,

ZHANG WEI,

YANG FAN,

WANG JIAN,

WANG WEI,

ZHOU XI,

ZHANG TAO,

ZHANG LEI,
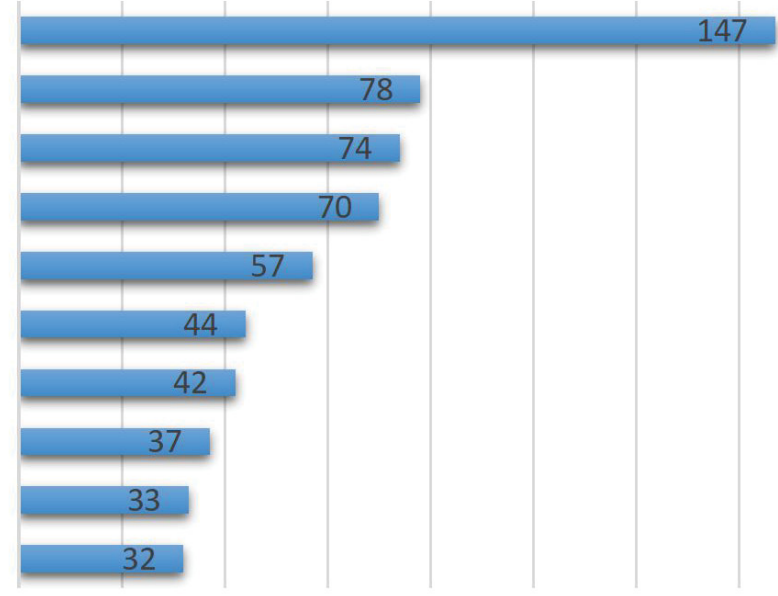

Fonte: Elaborado pelos autores deste artigo baseado na ferramenta Orbit (2019)

\subsection{Depositantes}

O Gráfico 4 mostra as 10 maiores empresas depositantes de patentes.

Gráfico 4 - As maiores depositantes de patentes

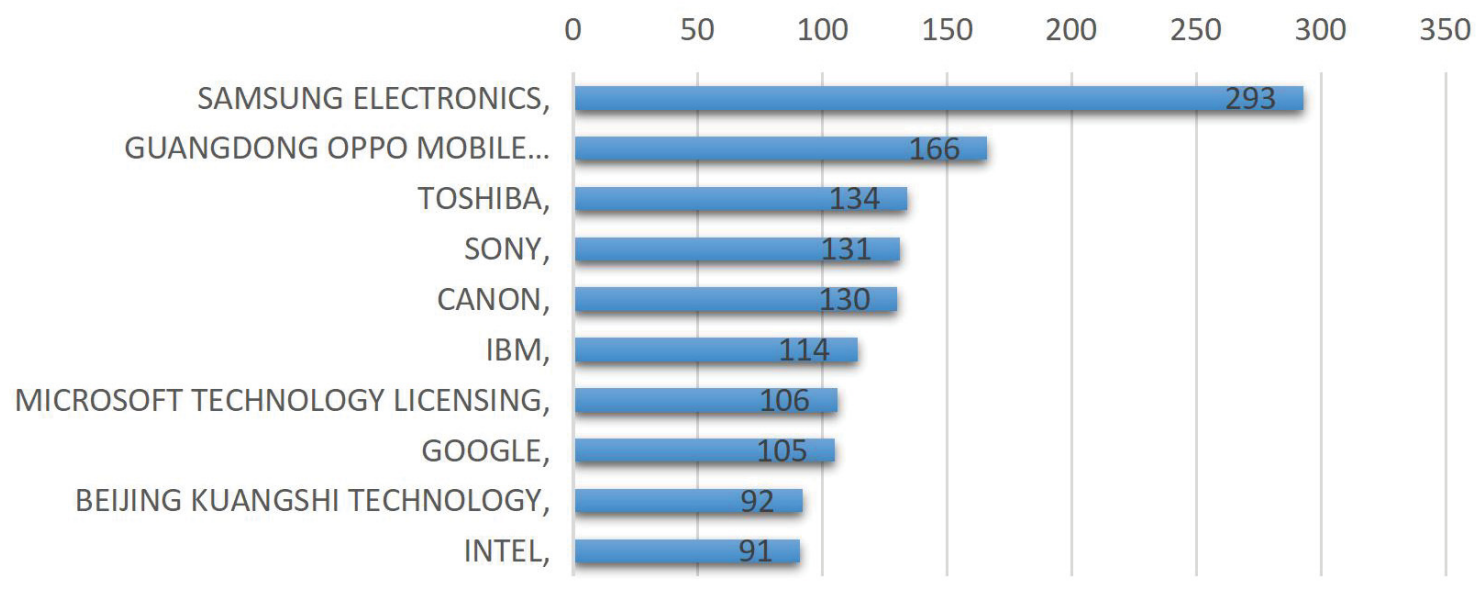

Fonte: Elaborado pelos autores deste artigo com base na ferramenta Orbit (2019)

Os principais depositantes são a Samsung, com 11,17\% dos registros, seguida da Guangdong Oppo Mobile Telecommunications, com 6,33\% dos registros.

A Samsung é uma empresa coreana fundada em 1938 como uma loja de venda de secos e molhados e fazia importação e exportação de peixes desidratados, arroz e macarrão, porém, dez anos depois, tornou-se uma das maiores empresas desse setor no país. O nome é derivado da palavra "Sam" que significa três e "Sung" que significa estrela (SAMSUNG, 2018). 
Em 1969, tornou-se uma grande fabricante coreana de produtos eletrônicos, semicondutores, telecomunicações óticas, e de novos campos da nanotecnologia para arquiteturas avançadas da rede, iniciando a produção de televisão em preto e branco a partir de 1970, e, em 1971, começou a exportar para o Panamá. Em 1974, começou a fabricar geladeira e máquina de lavar. A partir de 1977, começou a produzir e exportar televisão colorida. Na década de 1980, entrou no mercado global, e, em 1988, lançou o primeiro celular. Na década de 1990, impôs grandes negócios de alta tecnologia, transformando-se numa força global no seu segmento de atuação. Na década de 2000, foi pioneira na era digital, com produtos competitivos e de constante inovação. Em 2009, iniciou a venda dos celulares da família Galaxy; em 2010, vendeu mais de 24 milhões de unidades ao redor do mundo; em 2012, tornou-se a maior fabricante de telefonia do mundo; e, em 2014, vendeu mais de 40 milhões de unidades. Em 2018, fundou sete centros globais de inteligência artificial (KLEINA, 2017; SAMSUNG, 2018).

A Guangdong Oppo Mobile Telecomunications foi fundada em 2004 e se tornou um dos maiores fabricantes de smartphones do mundo. É a empresa líder global de telefones com câmera dedicada. A empresa possui seis institutos de pesquisas e quatro centros de P\&D.

Os principais países dos depositantes são a China com 1.131, seguida dos Estados Unidos com 922 .

As tecnologias dominantes estão divididas em cinco grupos: Química, Instrumentos, Engenharia Elétrica, Engenharia Mecânica e Outros Campos. O grupo dominante é o da Engenharia Elétrica com 70,88\% das patentes como mostra o Gráfico 5. Dentro do grupo, o destaque é a tecnologia da computação com 11.411 patentes registradas.

Gráfico 5 - Grupo de tecnologia dominante

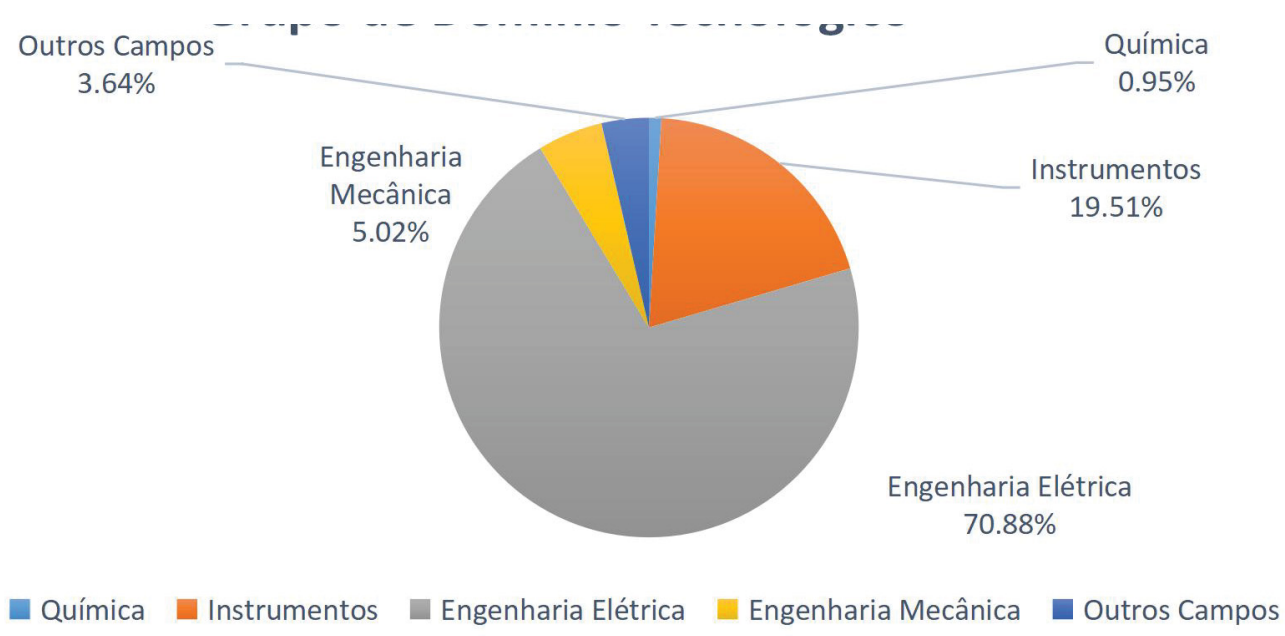

Fonte: Elaborado pelos autores deste artigo com base na ferramenta Orbit (2019)

As tecnologias decorrentes do computador podem se inserir nos avanços ocorridos com os hardwares (parte física do computador), assim como com os softwares (programas de computador). 


\section{Considerações Finais}

A tecnologia de IA está em um crescimento constante, principalmente como política pública de segurança, com o reconhecimento facial estando presente em 64 países, tendo a China como um dos principais usuários dessa tecnologia, que é utilizada para fins de vigilância. Também essa tecnologia está presente no dia a dia da população por meio dos celulares que, entre outras funções, selecionam as músicas e as atividades realizadas pelos usuários das redes sociais

A prospecção tecnológica tem um papel importante e essencial no desenvolvimento de projetos de cunho tecnológico, tornando-se obrigatório e fundamental o seu uso pela comunidade acadêmica e científica como um fator influenciador do processo de pesquisa e desenvolvimento tecnológico, no qual é possível observar a anterioridade, avaliar a viabilidade econômica, além de identificar as tendências tecnológicas e as formas de redução do tempo no processo de inovação, as oportunidades e os elementos necessários para o desenvolvimento da pesquisa.

Esta pesquisa apontou o maior número de patentes classificadas como IPC G06K que objetiva a identificação dos caracteres, como é o caso do reconhecimento facial, que utiliza como parâmetro a geometria da face. Outro código que também se sobressaiu foi o G06F, que diz respeito aos sistemas computacionais baseados em modelos computacionais específicos utilizando como meio a manipulação e o processamento dos dados. Esses dados apontam uma associação da ferramenta de reconhecimento facial para a criação de máquinas inteligentes, que pode, por exemplo, ser utilizada para segurança pública na identificação de pessoas, estando também presente no nosso cotidiano, principalmente nos celulares e nas redes sociais.

Os dados apresentados mostraram o cenário para um crescente investimento na área de tecnologias para o reconhecimento facial, o que representa um nicho de pesquisa e de mercado para instituições públicas e privadas.

Países desenvolvidos são os mais destacados como depositantes dessa tecnologia, o que mostra a importância de se investir nas áreas de pesquisa e de inovação para o crescimento econômico e para o desenvolvimento social.

Esse meio sistemático de prospecção constitui ferramenta importante para a evolução tecnológica no presente e no futuro, sendo uma técnica eficiente na utilização e na identificação de pessoas por meio do reconhecimento facial em locais de acessibilidade pública.

\section{Referências}

ACQUISTI, Alessandro; GROSS, Ralph; STUTZMAN, Fred. Face Recognition and Privacy in the Age of Augmented Reality. Journal of Privacy and Confidentiality, [S.I.], v. 6, n. 2, p. 1-20, 2014. Disponível em: https://jbit.ly/3fqQm5h. Acesso em: 10 jul. 2019.

ALVES, J. B. da Mata. Inteligência artificial: Aula 2. 2000. Disponível em: https://bit.ly/2z9xkjn. Acesso em: 29 jun. 2019.

ALVES, J. B. da Mata; CARUSO, L. A. C.; TIGRE P. B. Modelo SENAI de prospecção: documento metodológico. Montevideo: OIT/Cinterfor, Capítulo 2: Prospecção Tecnológica. (Papeles de la oficina técnica, 14). 2004. 
BATISTA, Gabriel Almeida et al. Sistema de identificação e autenticação biométrica. 2017. 121f. Atividade supervisionada curso Ciências da Computação - Universidade Paulista, São Paulo, 2017. Disponível em: https://bit.ly/2WxeMle. Acesso em: 26 jun. 2019.

BORINI, Guilherme. 11 formas como a inteligência artificial já faz parte do cotidiano. 2017. Disponivel em: https://bit.ly/2WzXkN3. Acesso em: 26 jun. 2019.

BRASIL. Ministério da Economia. Sistema de reconhecimento facial. [2019]. Disponível em: http://bit.ly/3ba17Wi. Acesso em: 26 jun. 2019.

COSSETI, Melissa Cruz. O que é inteligência artificial? 2018. Disponível em: https://bit. ly/2xH7seo. Acesso em: 27 jun. 2019.

DAVENPORT, Thomas et al. How artificial intelligence will change the future of marketing. Journal of the Academy of Marketing Science, [S.l.], p. 1-19, 2019. Disponível em: https://doi. org/10.1007/s11747-019-00696-0. Acesso em: 2 nov. 2019.

ELOLA. Joseba. O reconhecimento facial abre caminho para o pesadelo de George Orwell. 2018. Disponível em: https://bit.ly/2SBJzfq. Acesso em: 10 jul. 2019.

ESPACENET. Família de patentes. [2019]. Disponível em: https://bit.ly/2SEEH9u. Acesso em: 26 jun. 2019.

FELDSTEIN, Steven. The global expansion of AI surveillance. Carnegie Endowment for International Peace. 2019. Disponível em: https://bit.ly/3b5DZIv. Acesso em: 15 nov. 2019.

FIGLIUZZI, Renan Silva. Inteligência artificial: um novo paradigma tecnológico? 2018. 47f. Monografia (Bacharel em Economia) - Universidade Federal do Rio de Janeiro, Rio de Janeiro, 2018. Disponível em: https://bit.ly/2W7pQq3. Acesso em: 26 jun. 2019.

GIL, A. C. Como Elaborar Projetos de Pesquisa. 5. ed. São Paulo: Atlas, 2010.

INPI - INSTITUTO NACIONAL DA PROPRIEDADE INDUSTRIAL. Classificação de patentes. [2019]. Disponível em: http://bit.ly/3dm7B69. Acesso em: 26 jun. 2019.

KLEINA, Nilton. A história da Samsung, a gigante que veio da Coréia do Sul. 2017. Disponível em: https://bit.ly/3dk7lz1. Acesso em: 10 jul. 2019.

$\mathrm{KOCH}$, Márcio. Visão Computacional para Reconhecimento de Faces aplicado na Identificação e Autenticação de Usuários na Web. 2012. 139f. Trabalho de Conclusão do Curso (Bacharel em Ciência da Computação) - Universidade Regional de Blumenau, Blumenau, 2012. Disponível em: http:/bit.ly/2A3U817. Acesso em: 26 jun. 2019.

LAKATOS, E. M.; MARCONI, M. A. Metodologia do trabalho científico. São Paulo: Atlas, 1991.

MAYERHOFF, Zea Duque Vieira Luna. Uma análise sobre os estudos de prospecção tecnológica. Cadernos de Prospecção, Salvador, v. 1, n. 1, p. 7-9, 2008. Disponível em: https://bit.ly/2LOyZKK. Acesso em: 26 jun. 2020.

ORBIT. Plataforma Questel Orbit. Axonal. 2019. Disponível em: https://www.axonal.com.br/ capacitacao_info.php?id=114. Acesso em: 26 jun. 2019.

PORFÍRIO, Alexandre. Inteligência artificial estimula criação de novos negócios e acelera indústria 4.0. 2018. Disponível em: https://bit.ly/2YBuEpq. Acesso em: 19 jun. 2019. 
PRIESNITZ, Mariane Camargo et al. Prospecção da produção tecnológica da Fisioterapia. Mostra de Iniciação Científica, Pós-graduação, Pesquisa e Extensão, 15, 2015. Disponível em: http:// bit.ly/3b8WGek. Acesso em: 19 jun. 2019.

RIBEIRO, Núbia Moura (org.). Prospecção tecnológica. Salvador: IFBA, 2018. Disponível em: http://bit.ly/2yuW31uf. Acesso em: 19 jun. 2019.

RUSSEL, S.; NORVIG, P. Artificial intelligence: a modern approach. [S.l.]: Prentice-Hall, 1995.

SAMSUNG. Samsung aumenta investimento para crescimento futuro e toma iniciativa para construir ecossistema de inovação. 2018. Disponível em: https://bit.ly/2W8Wvvi. Acesso em: 26 jun. 2019.

SANTOS, Givaldo Almeida dos et al. Internet of Things (IoT): um cenário guiado por patentes industriais. Gestão Org., Edição especial, p. 271-281, 2015. Disponível em: https://bit.ly/2xCeZej. Acesso em: 26 jun. 2019.

VETTORAZZO, Lucas; PITOMBO, João Pedro. Rio e Salvador terão sistema de reconhecimento facial no carnaval. Folha de São Paulo, 2019. Disponível em: https://bit.ly/2YEHtiU. Acesso em: 26 jun. 2019.

WELCHEN. Vandoir. Uso de inteligência artificial em apoio a decisão clínica: o caso do Hospital do Câncer Mãe de Deus com a ferramenta cognitiva Watson on Oncology. 2019. $218 f$. Dissertação (Mestrado em Administração) - Universidade de Caxias do Sul, Caxias do Sul, 2019. Disponível em: https://it.ly/2SDHcJ0. Acesso em: 21 jun. 2019.

WIPO - WORLD INTELLECTUAL PROPERTY ORGANIZATION. Classificação Internacional de Patentes. 2019. Disponível em: http://bit.ly/3diap4f. Acesso em: 19 jun. 2019.

\title{
Sobre os Autores
}

\author{
Valdir Silva Conceição \\ E-mail: valdirconceicao@gmail.com \\ Mestrando do Programa de Pós-Graduação em Propriedade Intelectual e Transferência de Tecnologia para a \\ Inovação. \\ Endereço profissional: Rua Barão de Jeremoabo, n. 147, Ondina, Salvador, BA. CEP: 40170-115.
}

\section{Edna Maria Nunes}

E-mail: ednamnunes@hotmail.com

Mestranda do Programa de Pós-Graduação em Propriedade Intelectual e Transferência de Tecnologia para a Inovação.

Endereço profissional: Rua Barão de Jeremoabo, n. 147, Ondina, Salvador, BA. CEP: 40170-115.

\section{Angela Machado Rocha}

E-mail: anmach@gmail.com

Professora do Mestrado do Programa de Pós-Graduação em Propriedade Intelectual e Transferência de Tecnologia para a Inovação.

Endereço profissional: Rua Barão de Jeremoabo, n. 147, Ondina, Salvador, BA. CEP: 40170-115. 